NATURE, ORIGIN AND EVOLUTION OF INTERPLANETARY DUST GRAINS

\author{
Philippe L. Lamy \\ Laboratoire d'Astronomie Spatiale, Marseille
}

ABSTRACT: This contribution will attempt to give a coherent and up-todate view of the interplanetary dust cloud as well as to delineate some of the current outstanding problems. It is largely based on studies carried out at Laboratoire d'Astronomie Spatiale during the past years.

1. THE SIZE DISTRIBUTION AND PHYSICAL PROPERTIES OF INTERPLANETARY DUST GRAINS

Following years of inference of the size distribution of interplanetary dust grains from Zodiacal Light and F-Corona observations, the analysis of lunar microcraters provided a new and more direct means of solving this problem. Le Sergeant d'Hendecourt and Lamy (1980a) have carried out the most recent and comprehensive synthesis and thoroughly discussed all steps of the reduction process. The strong points of our analysis in comparison with the previous work of Fechtig et al. (1975) are the careful selection of well-documented "superior" samples in the production stage (no saturation) free of accretionary particles. Our final result appears in Fig. 1 where data biased by a lack of resolution has been removed. Fig. 1 strongly indicates the presence of two populations of interplanetary dust as was first proposed by Brownlee et al. (1975). In terms of a power law of the grain's radius $s \quad n(s)=n_{0} \cdot s-p$ for the differential spatial density at $1 \mathrm{AU}$, we observe a fairly constant population index for submillimetric grains $(p \simeq 3.95$ ) decreasing steadily with decreasing radii $(p \rightarrow 0)$; the inflection point signals the second population of submicronic grains having a constant population index $\mathrm{p}=3.85$ (Fig. 2).

It has been of concern that the large number of submicron-sized craters (for instance, in comparison with the results of Fechtig et al. 1975) could be due to a large contribution of secondary and even tertiary microcraters produced by ejecta from the primary ones. This question has been examined by Flavill et al. (1978) who showed that, for sample 12054 which was retained as the cornerstone of our analysis, the secondary population of microcraters could only exceed the primary distribution over the crater diameter range $1-10 \mu \mathrm{m}$. Their attempt to remove this 
"parasitic" contribution and to obtain the "derived primary microcrater distribution" is illustrated in Fig. 1 (dashed line) and translates into a displacement of the inflection point (from 6 to $3 \mu \mathrm{m}$ ) and a steepening of the distribution below this point implying a population index $\mathrm{p} \cong 4.6$. clearly, ths is not an argument to explain the discrepancy between our results and that of Fechtig et al. (1975) as the influence of secondary microcraters just goes in the opposite direction (steepening instead of flattening of the curve). Moreover, results for the smallest microcraters $(<0.2 \mu \mathrm{m})$ are not modified by the deconvolution process of Flavill et al. (1978) in agreement with the data from sample 76015, 24, 9003 which, because of its peculiar exposure geometry, was protected from secondary ejecta (Morrison and Clanton, 1979). Here again, it should be emphasized that these data were not normalized, (i.e., adjusted to the other samples) but are absolute values only corrected for the known exposure geometry.

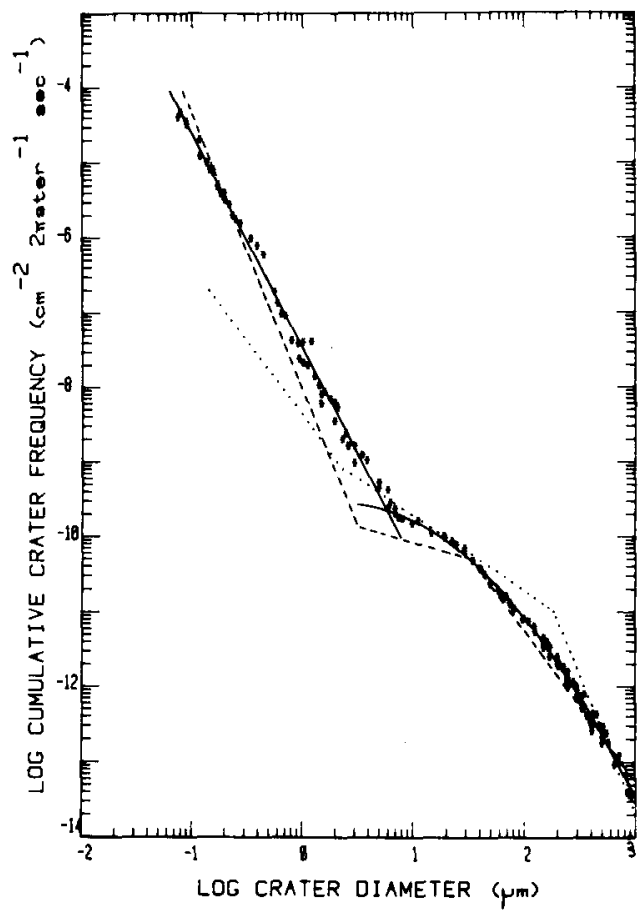

Fig. 1: The cumulative microcrater size distribution. The solid line represents our best fit, the dashed line the "derived primary microcrater distribution" of Flavill et a1. (1978) and the dotted line, the result of Fechtig et al. (1975).

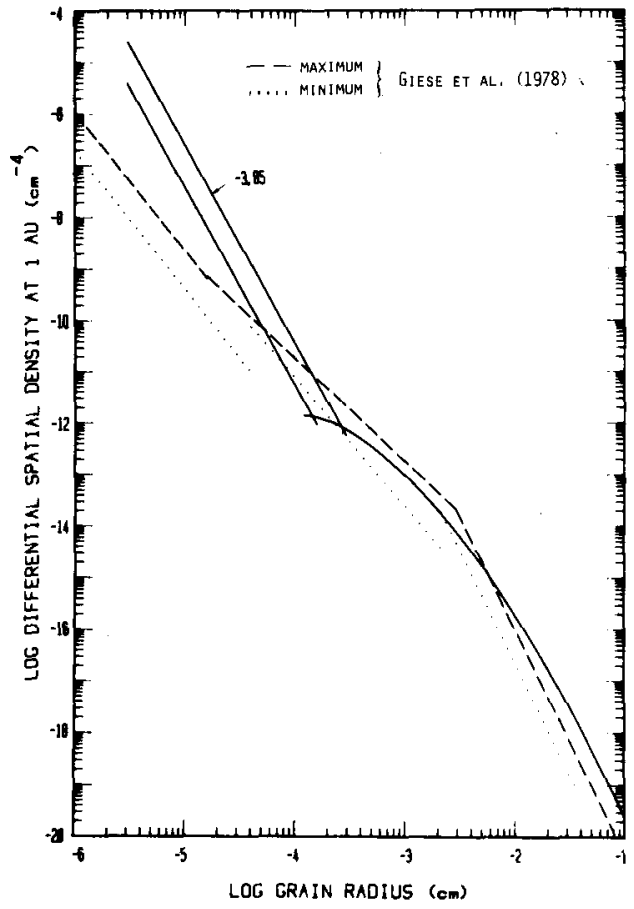

Fig. 2: The differential spatial density at $1 \mathrm{AU}$ (solid lines); as explained in the text, the upper curve should be favored. The maximum and minimum models of Giese et al. (1978) are shown for comparison. 
The differential spatial density at $1 \mathrm{AU}$ (Fig. 2) depends on the impact velocity of the grains, both directly and indirectly via the ratio Dc/d of the crater diameter to grain diameter. Contrary to the large grains, the situation is unclear for the submicronic grains and we have considered two cases $17 \mathrm{~km} / \mathrm{sec}$ (as for the 1arge grains) and $35 \mathrm{~km} / \mathrm{sec}$. The first solution (upper curve in Fig. 2) should be favored since it is supported both qualitatively (apex population, see below) and quantitatively by the Heos 2 and Pioneers 8 and 9 dust experiments (see Fig. 14 of Le Sergeant d'Hendecourt and Lamy, 1980a).

In the same article, we have shown how the two populations may be further differentiated on the basis of their bulk density: a detailed analysis of the depth to the diameter of 284 microcraters ( $P / D C$ ) revealed that the population "1" of large grains $(\mathrm{d}>2 \mu \mathrm{m})$ has a density typical of silicates, while the population " 2 " of small grains (d<2 $2 \mu \mathrm{m}$ ) is characterized by a higher density - possibly as high as that of iron with a minor component of silicates. Furthermore, low-density grains $\left(<1 \mathrm{~g} / \mathrm{cm}^{3}\right)$ were not detected. We pointed out the excellent agreement with the two populations which appear in dust grains collected in the Earth's upper atmosphere (Brownlee et al. 1976a, b):

- large compact aggregates having chondritic abundances and a mean density of $2 \mathrm{~g} / \mathrm{cm}^{3}$;

- smaller homogeneous, nearly spherical "iron-sulfur-nickel" (FSN) grains whose composition is consistent with troilite, containing 1 to $5 \%$ of nickel.

\section{COLLISIONS AMONG INTERPLANETARY DUST GRAINS}

It has been proposed (Zook and Berg, 1975; Dohnanyi, 1976) that the population " 2 " of small, essentially submicronic, grains could be the fragments of collisions between large grains ejected by radiation pressure thus creating an outflow of so-called "B-meteoroids" as detected by Pioneer 8 and 9 (Berg and Griln, 1973). Le Sergeant d'Hendecourt and Lamy (1981) have carefully examined this mechnism and concluded that the calculated flux of fragments could not match by far the observed flux of submicronic grains. The $B$-meteoroids present numerous difficulties which we have already discussed. In particular, since the population "1" of large grains is certainly composed of silicates, so are the fragments and radiation pressure will not allow them to suffer hyperbolic escape, except in a very narrow size range $(0.1$ to $0.4 \mu \mathrm{m})$. It is revealing to note that this rather embarrassing component has been pushed to smaller and smaller radii or masses as time goes on: initially, Berg and Grun (1973) favored a mean mass of $10^{-12} \mathrm{~g}$ ( $\mathrm{d} \cong 1 \mu \mathrm{m}$ for silicates); McDonnell (1978) considered this value as an upper boundary and finally, Griln and Zook (1980) pushed this upper limit for $\beta$-meteoroids down to $10^{-14} \mathrm{~g}$.

We have proposed that $\beta$-meteoroids may possibly be a transitory phenomenon connected with solar or cometary activity. Indeed, Sekanina (1982) has shown that the surviving debris of Comet Howard-Koomen-Michels 
(1979 XI) which collided with the Sun consisted mostly of absorbing, submicron-size grains in hyperbolic trajectories convex to the Sun and curving toward the Earth. As this kind of catastrophic event appears to occur quite often, we possibly have a sound basis for explaining the erratic behaviour of $\beta$-meteoroids. Another important consequence is that the population "2" of submicronic grains needs not to be directly related to $\beta$-meteoroids and therefore, there is no reason to impose large hyperbolic orbital velocities for these grains.

Our collisional model has also helped us to reach several other interesting conclusions. Grains larger than $100 \mu \mathrm{m}$ are destroyed by catastrophic collisions inside 1 AU. Contrary to a common view, this process is not localized near the Sun but takes place rather uniformly inside the 1 AU sphere (and probably outside too). Henceforth, their lifetime is not controlled by the Poynting-Robertson effect, but instead by catastrophic collisions. Likewise, the production of fragments is not localized in a small region near the Sun but is substantial throughout the $1 \mathrm{AU}$ sphere. For example, the regions $0.02-0.25$ and $0.25-1 \mathrm{AU}$ equally contribute to the flux of fragments.

\section{IMPLICATIONS FOR THE ZODIACAL LIGHT}

Preliminary implications of the above results for the Zodiacal Light have been examined by Lamy and Perrin (1980). Let us further analyze the various constraints. First, our differential spatial density of Fig. 2 was directly obtained as a function of grain radius and not of mass; therefore, we could not play with the bulk density of grains, as Giese et a1. (1978) did, to artificially increase the grain's radius for a given mass, resulting in a substantial increase of the scattered 1 ight. Second, the albedo of the grains is constrained by the dark appearance of collected micrometeorites and the infrared brightness of the Zodiacal Light (Hanner 1980). Based on the similarity between these collected grains and the carbonaceous chondrites, a value of the albedo $p=0.05$ as determined for the latter by Johnson and Fanale (1973) seems realistic. From microwave analog measurements of a slightly absorbing "fluffy" particle, Hanner (1981) deduced an albedo of $\mathrm{p}=0.055$ at medium scattering angles in good agreement with the above value. Under these constraints, Lamy and Perrin (1980) have shown that the population "1" of large grains alone cannot explain the observed volume scattering function of the Zodiacal Light (Fig. 3). Indeed, in order to obtain this agreement with their maximum model which is close to ours in this size range, Giese and Grln (1976) implicitly used an albedo of 0.24 as given by Hanner (1980). We have demonstrated how the enhancement in the scattering intensity observed on rough, but relatively smal1, particles by Giese et al. (1978) improved the situation. However, recent measurements by Weiss (private communication) on larger particles more appropriate to the Zodiacal Light problem reveal a more modest enhancement.

In view of the above problem, we have pointed out that the population " 2 " of small grains presents a non-negligible contribution to the 
volume scattering function and may well account for the above discrepancy, a view supported by Hanner (1981). The exact contribution cannot be specified at this early stage of investigation. It strongly depends on the properties of the grains. We have fully realized that the neutral color of the Zodiacal Light as well as its polarization indicate that the submicronic grains cannot dominate the optical scattering process. This important point has been emphasized by Hanner (1981). On the contrary, Leinert et al. (1981, 1982) have repeatedly distorted our conclusions, implying that we were advocating a "dominating population of small particles in interplanetary space". On the basis of the calculations of Lamy and Perrin (1980) and our present knowledge of interplanetary dust, a good indication may be obtained from the "fluffy" model for population 1 and pyrrhotite for population 2 (Fig. 3). This nonstoichiometric iron sulfur is probably representative of the FSN particles of Brownlee. Then, the large grains contribute to $2 / 3$ of the volume scattering function while the small ones contribute to: $1 / 3$ approximately. The sum of the two contributions matches the observations. Once again, the models were too crude to extract more information than they really contain. Perrin and Lamy (in preparation) have established a new model of light scattering by large, rough grains and are presently re-investigating this question.

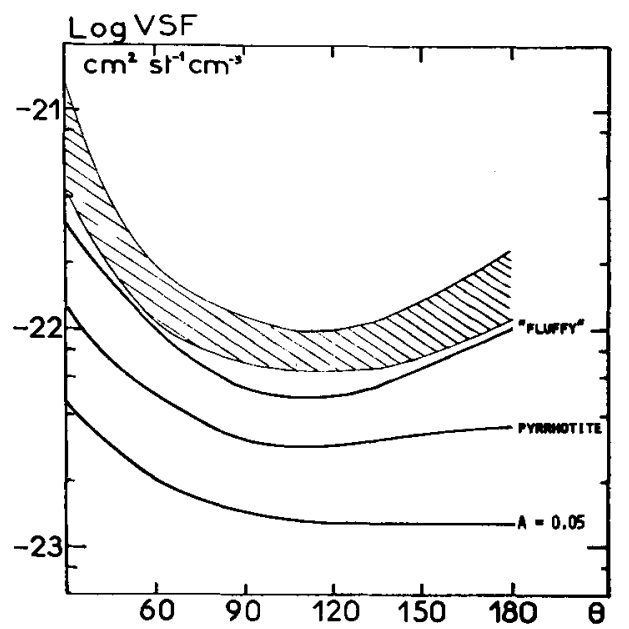

Fig. 3: Comparison of the range of observed Volume Scattering Function (grey band) and different models: Population 1: single Fresnel reflection + isotropic term with albedo $A=0.05$ and "fluffy"

Population 2: pyrrhotite.

Leinert et a1. (1981) have been amazed that the effects of collisions between interplanetary grains did not appear in the Helios data. A simple examination of the results of Le Sergeant d'Hendecourt and Lamy (1981) allows one to understand this situation. From the ratio of the flux of collisional fragments to that actually observed (our Fig. 1) and from the above discussion of the contribution to the volume scattering function, we conclude that these fragments will account for less than $0.16 \%$ of the Zodiacal Light, a quantity which is not detectable.

\section{MASS FLUX AT 1 AU}

Le Sergeant d'Hendecourt and Lamy $(1978,1980 \mathrm{~b})$ have already made comments on this question based on preliminary analysis. Our estimates 
can now be refined using our final results (1980a, 1981).

i) Population 1 mass flux: the Poynting-Robertson effect creates an inward mass flux whivh has been shown to be

$$
\mathrm{F}_{1}=4.0 \times 10^{-22} \mathrm{~g} \mathrm{~cm}^{-2} \mathrm{sec}^{-1}
$$

in the ecliptic plane. Taking into account a proper distribution of inclinations, the total mass crossing the $1 \mathrm{AU}$ sphere per unit time is

$$
\dot{\mathrm{M}}_{1}=2.2 \times 10^{5} \mathrm{~g} \mathrm{sec}^{-1}
$$

Sekanina and Schuster (1978a, b) have shown that short-period comets d'Arrest and Encke have the product of grain albedo and production rate of micrometeoroids averaged over the orbit equal to $200 \mathrm{~g} \mathrm{sec}^{-1}$. Retaining an albedo of 0.05 as justified above, 55 short-period comets similar to d'Arrest and Encke are required to maintain population 1 in steady state. This is just the number of periodic comets of more than one appearance having periods less than 20 years (Marsden 1975). Furthermore, the size range (for a bulk density of $2 \mathrm{~g} / \mathrm{cm}^{3}$ ) and the exponent of the size distribution deduced by Sekanina and Schuster agree surprisingly well with that of pupulation 1.

ii) Population 2 mass flux: as a first case, let us consider that this population is composed of $\beta$-meteoroids leaving the solar system on hyperbolic orbits. Then, the mass loss rate through the $1 \mathrm{AU}$ sphere can be shown to be

$$
\dot{\mathrm{M}}_{2}=1.5 \text { to } 2.3 \times 10^{8} \mathrm{~g} \mathrm{sec}^{-1}
$$

(depending upon the choice of velocity), that is 1000 times the value found for population 1 . This quantity represents a formidable challenge as Le Sergeant d'Hendecourt and Lamy (1978) already pointed out. But, we have seen that this assumption is probably not correct and that population 2 grains most likely move in bound orbits. Then the mass loss rate through the 1 AU sphere under the Poynting-Robertson effect can be calculated as in population 1 . It is found to be:

$$
\dot{\mathrm{M}}_{2}=8.3 \times 10^{5} \mathrm{~g} \mathrm{sec}^{-1}
$$

that is approximately four times that of population 1. It is not out of question that short-period comets could also provide this quantity of submicronic grains since these grains may escape detection. This hypothesis requires further examination.

\section{REFERENCES}

Berg, O.E., Grlin, E.: 1973, COSPAR, 15th Meeting, In Space Research XIII, pp. 1047-1056. Akademie-Verlag, Berlin.

Brownlee, D.E., Hörz, F., Hartung, J.C., Gault, D.E.: 1975, Proc. Lunar Sci. Conf. 6th, pp. 3409-3416. 
Brownlee, D.E., Tomand1, D.A., Hodge, P.W.: 1976a, IAU Colloquium No. 31, Lecture Notes in Physics, No. 48, pp. 279-283. Springer-Verlag, Berlin.

Brownlee, D.E., Hörz, F., Tomand1, D.A., Hodge, P.W.: 1976b, IAU Co11oquium No. 25, pp. 962-981. NASA SP-393.

Dohnanyi, J.S.: 1976, Interplanetary Dust and Zodiacal Light (H. Elsässer and H. Fechtig, Eds), pp. 170-180, Springer-Verlag Ber1in.

Fechtig, H., Gentner, W., Hartung, J.B., Nage1, K., Neukum, G., Schneider, D., Storzer, D.: 1975, Proceedings of the Soviet-American Conference Cosmochemistry, Moscow, 1974.

Flavil1, R.P., Allison, R.J., McDonne11, J.A.M.: 1978, Proc. Lunar Planet. Sci. Conf. 9th, pp. 2539-2556.

Giese, R.M., Grltn, E.: 1976, Lect. Notes in Physics 48, pp. 135-139.

Giese, R.M., Weiss, K., Zerul1, R.H., Ono, T.: 1978, Astron. Astrophys. 65, pp. 265-270.

GrUn, E., Zook, H.A.: 1980, Solid Particles in the Solar System (I. Halliday and B. McIntosh, Eds), pp. 293-298.

Hanner, M.S.: 1980, Icarus 43, pp. 373-380.

Hanner, M.S.: 1981, Astron. Astrophys. 104, pp. 42-46.

Johnson, T.V., Fanale, F.P.; 1973, J. Geophys. Res. 78, pp. 8507-8518.

Lamy, P.L., Perrin, J.M.: 1980, Solid Particles in the Solar System (I. Halliday and B.McIntosh, Eds), pp. 75-80.

Leinert, C., Richter, I., Pitz, E., P1anck, B.: 1981, Astron. Astrophys. 103, pp. 177-188.

Leinert, C., Richter, I., Planck, B.: 1982, Astron. Astrophys. 110, pp. 111-114.

Le Sergeant d'Hendecourt, L., Lamy, P.L.: 1978, Nature 276, pp. 800-802.

Le Sergeant d'Hendecourt, L., Lamy, P.L.: 1980a, Icarus 43, pp. 350-372.

Le Sergeant d'Hendecourt, L., Lamy, P.L.: 1980b, Solid Particles in the Solar System (I. Halliday and B. McIntosh, Eds), pp. 289-292.

Le Sergeant d'Hendecourt, L., Lamy, P.I.: 1981, Icarus 47, pp. 270-281. Marsden, B.G.: 1975, Catalogue of Cometary Orbits (2nd ed.), IAU Central Bureau for Astronomical Telegrams.

McDonne11, J.A.M.: 1978, in J.A.M. McDonnel1 (ed.), Cosmic Dust, J.Wiley and Sons, Chichester, Ch. 6, pp. 337-426.

Morrison, D.A., Clanton, U.S.: 1979, Proc. Lunar Planet. Sci. Conf. 10th, pp. 1649-1663.

Sekanina, Z., Schuster, H.E.: 1978a, Astron. Astrophys. 65, pp. 29-35.

Sekanina, Z., Schuster, H.E.: 1978b, Astron. Astrophys. 68, pp. 429-435.

Sekanina, Z.: 1982, Astron. J. 87, pp. 1059-1072.

Zook, H.A., Berg, O.E.: 1975, Planet. Space Sci. 23, pp. 183-203.

\section{DISCUSSION}

FECHTIG: 1) Submicron-sized lunar craters can be "contaminated" by secondary craters. 2) Recent calculations by E. Grin, Heidelberg show that the so-called $\beta$-meteoroids are produced by larger meteoroids through collisional processes within $1 \mathrm{AU}$.

LAMY: 1) Attempt by Flavill et al. (1978) to remove this effect resulted in a steepening of the curve (see above discussion). 2) I look forward 
to examine this calculation.

WHIPPLE: It is very satisfying to see the confirmation of my calculations of the $1950^{\prime} \mathrm{s}$, i.e. the order of 1 to $10 \times 10^{6} \mathrm{~g} \mathrm{sec}^{-1}$ for input mass rate.

LAMY: The total mass loss rate for the two populations (assuming population 2 in bound orbits) amounts to $1.05 \times 10^{6} \mathrm{~g} \mathrm{sec}^{-1}$. Our direct calculation is indeed in good agreement with your indirect method. If we consider only the grains which contribute to the Zodiacal Light (cf. your method), the mass loss rate would be slightly smaller. 